\title{
Role of the BaeSR two-component system in the regulation of Acinetobacter baumannii ade $A B$ genes and its correlation with tigecycline susceptibility
}

\author{
Ming-Feng Lin ${ }^{1,2}$, Yun-You Lin ${ }^{1}$, Hui-Wen Yeh ${ }^{3}$ and Chung-Yu Lan ${ }^{2,4^{*}}$
}

\begin{abstract}
Background: Tigecycline resistance in Acinetobacter baumannii is primarily acquired through overexpression of the AdeABC efflux pump. Besides AdeRS, other two-component regulatory systems (TCSs) involving the regulation of this transporter have not been clarified.

Results: In this study, we found that the TCS genes baeR and baeS are co-transcribed and function as stress responders under high osmotic conditions. The baeSR and adeAB genes showed increased transcription in both the laboratory-induced and clinical tigecycline-resistant strains compared with the wild-type strain. The deletion of baeR in the ATCC 17978 strain led to 67-73\% and 68\% reduction in adeA and adeB expression, respectively, with a resultant 2 -fold decrease in the tigecycline minimal inhibition concentration (MIC). In contrast, the overexpression of baeR resulted in a doubled tigecycline MIC, with a more than 2 -fold increase in adeA and adeB expression. The influence of baeR knockout on adeAB gene expression can also be observed in the laboratory-induced tigecycline-resistant strain. A time-kill assay showed that the baeR deletion mutant showed an approximate $1-\log _{10}$ reduction in colony forming units (CFUs) relative to the wild-type strain when the tigecycline concentration was $0.25 \mu \mathrm{g} / \mathrm{mL}$ throughout the assay period. The wild-type phenotype could be restored by trans-complementation with pWH1266-kan'-baeR. Increasing the tigecycline concentration to $0.5 \mu \mathrm{g} / \mathrm{mL}$ produced an even more marked $4.7-\log _{10}$ reduction in CFUs of the baeR deletion mutant at $8 \mathrm{~h}$, while only a $2.1-\log _{10}$ reduction was observed for the wild-type strain.
\end{abstract}

Conclusions: Taken together, these data show for the first time that the BaeSR TCS influences the tigecycline susceptibility of A. baumannii through the positive regulation of the resistance-nodulation-division efflux pump genes adeA and adeB.

Keywords: Acinetobacter baumannii, Tigecycline, Two-component regulatory system, Efflux pumps

\section{Background}

Acinetobacter baumannii has emerged as a major cause of nosocomial infections, especially in intensive care units [1]. Both its ability to acquire resistant determinants and to adapt to harsh environments has made $A$. baumannii a successful pathogen [2]. A. baumannii has high rates of resistance to many available antibiotics in clinical practice.

\footnotetext{
* Correspondence: cylan@life.nthu.edu.tw

${ }^{2}$ Institute of Molecular and Cellular Biology, National Tsing Hua University, Hsin-Chu City, Taiwan

${ }^{4}$ Department of Life Science, National Tsing Hua University, Hsin-Chu City, Taiwan

Full list of author information is available at the end of the article
}

For example, imipenem-resistant $A$. baumannii constituted $>50 \%$ of a worldwide collection of clinical samples between 2005 and 2009 [3]. A Taiwanese surveillance report of antimicrobial resistance in 2000 found that $73 \%$ of A. baumannii isolates collected from 21 medical centers and regional hospitals were ceftazidime-resistant [4]. Therefore, there are only a few effective anti-Acinetobacter drugs currently available, including polymyxins and tigecycline [5]. Tigecycline is the first drug from the glycylcycline class, a new class of antibiotics derived from tetracycline [6]. Tigecycline acts as a protein synthesis inhibitor by binding to the $30 \mathrm{~S}$ ribosomal subunit, and thus

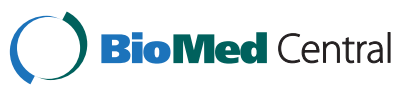

(c) 2014 Lin et al.; licensee BioMed Central Ltd. This is an Open Access article distributed under the terms of the Creative Commons Attribution License (http://creativecommons.org/licenses/by/2.0), which permits unrestricted use, distribution, and reproduction in any medium, provided the original work is properly credited. The Creative Commons Public Domain Dedication waiver (http://creativecommons.org/publicdomain/zero/1.0/) applies to the data made available in this article, unless otherwise stated. 
blocking entry of the tRNA into the A site of the ribosome during translation. Although tigecycline has an expanded spectrum of antibacterial activity, previous studies have shown that tigecycline resistance has emerged in $A$. baumannii. Resistance in these strains is associated with multidrug efflux systems, especially the overexpression of the ade $A B C$ genes, which encode an efflux pump $[7,8]$. The AdeABC pump belongs to the resistancenodulation-division (RND) family, which has a threecomponent structure [9].

Bacterial two-component systems (TCSs) play an important role in the regulation of adaptation to and signal transduction of environmental stimuli, including stress conditions [10]. TCSs are typically composed of a membrane-localized sensor with histidine kinase activity and a cytoplasmic response regulator (RR). Generally, upon sensing environmental changes, signaling begins via autophosphorylation of the sensor protein at a conserved histidine residue. The phosphate is then transferred to an aspartic acid residue in the so-called receiver domain of the corresponding RR. Phosphorylation may induce conformational changes in RRs, which alters their DNAbinding properties, thus modulating downstream gene expression [11]. Importantly, the roles of TCSs in the regulation of antimicrobial resistance have recently been documented in several species of bacteria [12-14]. Additionally, the AdeS-AdeR TCS controls genes encoding the AdeABC pump in A. baumannii [15]. AdeS is a sensor kinase, whereas AdeR is an RR. Point mutations in AdeS and AdeR, or a truncation of AdeS due to an ISAba1 insertion, may be related to the overexpression of AdeABC, which leads to multidrug resistance $[15,16]$. However, the existence of ade $A B C$-overexpressing mutants without any mutations in adeRS [7] and the low expression of adeABC in a clinical strain of $A$. baumannii with the ISAbaI insertion in the adeRS operon [16] suggest that the regulation of adeABC gene expression is complicated, and other regulatory mechanisms may be involved.

BaeSR is a TCS and is one of the five extracytoplasmic response pathways in Escherichia coli. BaeSR detects environmental signals and responds by altering the bacterial envelope [17]. The main function of the Bae response is to upregulate efflux pump expression in response to specific envelope-damaging agents [18]. Indole, flavonoids, and sodium tungstate have been shown to be novel inducers of the BaeSR response [18,19]. In Salmonella typhimurium, one of the physiological roles of BaeR is to respond to stresses that specifically damage MdtA, leading to an induction of MdtA transport and the removal of the toxic agent (e.g., tungstate waste) from the cell [19]. In TolC mutants or efflux mutants of $E$. coli, the overexpression of spy, which encodes a periplasmic chaperone, depends on the BaeRS and CpxARP stress response systems [20]. A genome-wide analysis of $E$. coli gene expression showed that BaeR overproduction activates genes involved in multidrug transport, flagellum biosynthesis, chemotaxis, and maltose transport [21]. Furthermore, BaeSR is also able to activate the transcription of the $\operatorname{yeg} M N O B$ ( $m d t A B C D$ ) transporter gene cluster in $E$. coli and increases its resistance to novobiocin and deoxycholate [22]. Because there is a potential similarity in the biological functions of $m d t A B C D$ in $E$. coli and adeABC in A. baumannii, we here explore the role of BaeSR in the regulation of the transporter gene ade $A B$ in $A$. baumannii and report the positive regulation of these factors, which leads to increased tigecycline resistance.

\section{Results}

\section{Sequence analysis of the $A d e A B$ efflux pump and the BaeR/Baes TCS}

A search of the GenBank database (http://www.ncbi.nlm. nih.gov/genbank) revealed that, similar to other strains of A. baumannii, the ATCC 17978 strain contains sequences encoding the AdeABC-type RND efflux pump. There are two adeA genes (A1S_1751 and A1S_1752) and one adeB gene (A1S_1750) in the genome; however, no adeC gene was found. $A d e B$ is a transmembrane component with two conserved domains: the hydrophobe/amphiphile efflux-1 (HAE1) family signature and a domain conserved within the protein export membrane protein SecD_SecF. Both AdeA proteins are inner membrane fusion proteins with biotin-lipoyl-like conserved domains. We designated A1S_1751 as AdeA1 and A1S_1752 as AdeA2 for differentiation.

The A. baumannii ATCC 17978 gene A1S_2883 encoded a protein of 228 amino acids. Sequence alignments of A. baumannii A1S_2883 with BaeR homologs in other bacteria showed that A1S_2883 shared 64.6\% similarity with BaeR of E. coli str. K-12 substr. MG1655 and 65.2\% similarity with BaeR of Salmonella enterica subsp. enterica serovar Typhimurium str. LT2 (Figure 1A). In addition, protein analysis using Prosite (http://prosite. expasy.org/) predicted that A. baumannii A1S_2883 contained a response regulatory domain at amino acid residues 3 to 115 and a phosphorylation site at amino acid residue 51 (aspartate). Therefore, the role of A1S_2883 may be similar to that of BaeR in other bacterial species; thus, we have designated A1S_2883 as BaeR in $A$. baumannii.

A. baumannii ATCC 17978 gene A1S_2884 encodes a protein of 487 amino acids. Sequence alignments showed that A1S_2884 shared 48.1\% similarity with BaeS of E. coli str. K-12 substr. MG1655 and 46.3\% similarity with BaeS of Salmonella enterica subsp. enterica serovar Typhimurium str. LT2 (Figure 1B). Protein analysis using Prosite predicted that $A$. baumannii A1S_2884 contains a HAMP (histidine kinase, adenylyl cyclase, methyl-accepting protein, and phosphatase) and a histidine kinase domain at 


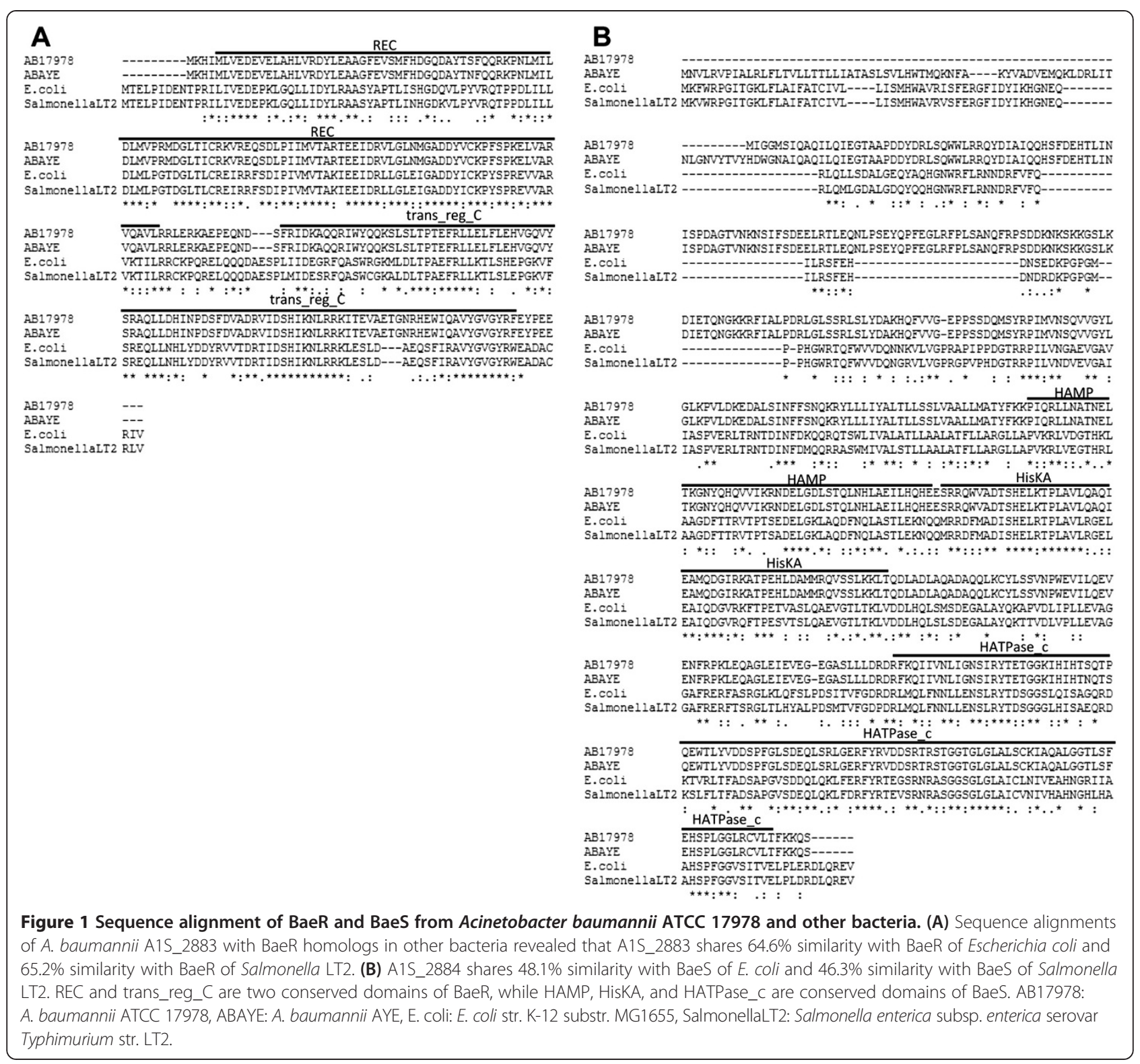

amino acid residues 214 to 266 and 274 to 487 , respectively. In addition, the histidine at residue 277 is predicted to be a phosphorylation site. Therefore, the role of A1S_2884 may be similar to that of BaeS in other bacterial species; thus, A1S_2884 is designated as BaeS in $A$. baumannii.

\section{Co-transcription of baeR and baes as an operon}

Although the BaeSR TCS has been characterized in $E$. coli [21,22], the biological functions of BaeSR in A. baumannii have not been revealed. Genome analysis of $A$. baumannii ATCC 17978 shows that the coding sequences of baeR (A1S_2883) and baeS (A1S_2884) are arranged sequentially, suggesting that the two genes may be co-transcribed as an operon. To test this hypothesis, reverse transcription-polymerase chain reaction (RT-PCR) was performed using the primers baeS-co F and baeS-co $\mathrm{R}$ (Table 1). As shown in Figure 2A, a 793-bp DNA fragment covering the junction between both baeR and baeS was amplified by RT-PCR. We concluded that baeR and baeS are co-transcribed as a single operon in A. baumannii ATCC 17978.

\section{Transcription of baeR and baeS under normal and stressed conditions}

TCSs are commonly involved in stress responses in bacteria. Because no previous studies have explored the 
Table 1 Oligonucleotides used in this study

\begin{tabular}{|c|c|}
\hline Primer name & Sequence $\left(5^{\prime} \text { to } 3^{\prime}\right)^{a}$ \\
\hline baeS-co_F & CGCGTAGTACAGGTGGAACA \\
\hline baeR-co_R & TCCACTCATGACGGTTACCA \\
\hline$k a n^{r}-$ BamHI_F & ATATGGATCCCCGGAATTGCCAGCTGGGGC \\
\hline$k a n^{r}-K p n l \_R$ & ATATGGTACCTCAGAAGAACTCGTCAAGAA \\
\hline baeR-up-Sall_F & TTAAGTCGACCGCCCGATTATGGTCAATAG \\
\hline baeR-up-BamHI_R & TATAGGATCCGCTTACTTCGAACCCAGCAG \\
\hline baeR-dw-Kpnl_F & ATCGGGTACCTTGCTTAGAAAAGTTATGCT \\
\hline baeR-dw-Sacl_R & AAATGAGCTCATGCTTTAGGGGTGGCTTCT \\
\hline baeR-up-check_R & CTTCCCAGTGGTGGTTACG \\
\hline baeR-dw-check_R & GACGGACGTGGCTTACTCAT \\
\hline pWH1266 check_F & TGCCACCTGACGTCTAAGAA \\
\hline pWH1266 check_R & TCATACACGGTGCCTGACTG \\
\hline$S a c B \_F$ & AGTTTTGTTCAGCGGCTTGT \\
\hline$S a c B \_R$ & GGTCAGGTTCAGCCACATTT \\
\hline baeR_F & GGATGGTTAAACGATTTGCC \\
\hline baeR_R & TCCACTCATGACGGTTACCA \\
\hline baes_F & CTTCCCAGTGGTGGTTACG \\
\hline baes_R & GAGCCAAGTCTGCCAAATCT \\
\hline baes probe_F & CCTCAATACTGGTGAAACCA \\
\hline baes probe_R & TCCCCCAATCATGATAAACG \\
\hline rpoB_F & AGTCACGCGAAGTTGAAGGT \\
\hline$r p o B \_R$ & GCGGTATGGAGTTTCCAAGA \\
\hline 16 s rRNA_F & GTAGCTTGCTACTGGACCTAG \\
\hline 16 s rRNA_R & CATACTCTAGCTCACCAGTATCG \\
\hline Kan-2'-EcoRI_F & AATAGAATTCACATCTCAACCATCATCG \\
\hline Kan-2'-EcoRI_R & AATAGAATTCCATCTCAACCCTGAAGC \\
\hline baeR-Xhol_F & TTAACTCGAGCATGTTTCATGATGGTC \\
\hline baeR-Xbal_R & TAGCTCTAGATTATTCTTCTGGATATTCG \\
\hline qbaes_F & GCCATTCAGCAGCATTCTITC \\
\hline qbaes_R & ATTAACCGTTCCCGCATCTG \\
\hline qbaeR_F & TGACAGCACGTACCGAAGAAA \\
\hline qbaeR_R & CATAATCATCTGCCCCCATGT \\
\hline qadeB_F & ACAAGACCGCGCTAACTTAGGT \\
\hline qadeB_R & TGCCATTGCCATAAGTTCATCT \\
\hline qadeA1_F & CCTCAAGCGCTATTGGTTCCT \\
\hline qadeA1_R & CCTGAGGCTCGCCACTGA \\
\hline qadeA2_F & TTGAGGCCGATGTAAATAGCA \\
\hline qadeA2_R & GTCTTGCCACCTCAGCTTCAG \\
\hline q16s rRNA_F & AGCATTTCGGATGGGAACTTTA \\
\hline q16s rRNA_R & GTCGTCCCCGCCTTCCT \\
\hline
\end{tabular}

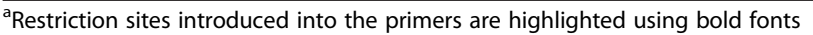
and described in the primer name. function of A1S_2883 and A1S_2884, we began by testing the response of both genes to high osmotic conditions to determine if they have functions that are similar to those of their BaeSR counterparts in other bacteria. To determine whether A. baumannii baeSR participates in the stress response, the relative levels of baeR and baeS transcription were detected in cells grown in LuriaBertani (LB) agar $\left(37^{\circ} \mathrm{C}, 220 \mathrm{rpm}\right)$ with or without $20 \%$ sucrose. RT-PCR analysis showed that the expression levels of baeR and baeS were 2.3- and 6.7-fold higher in cells exposed to osmotic stress compared with cells grown without sucrose (Figure 2B). This result suggested that the BaeSR TCS in A. baumannii was involved in cellular adaptation to stress conditions such as high osmolarity.

\section{Construction of baeR deletion mutants and baeR- reconstituted strains}

To further study the role of the BaeSR TCS in A. baumannii, in-frame deletion mutants of baeR were generated using the method of Sugawara et al. [23]. The successful construction of baeR deletion mutants was verified by PCR (Additional file 1: Figure S1B), RT-PCR (Additional file 2: Figure S2), and Southern blot assays (Additional file 3: Figure S3B). To generate the baeRreconstituted strain, pWH1266-kan ${ }^{r}-$ baeR was introduced into the baeR deletion mutant (AB1026; Table 2) by electroporation. The $b a e R$-reconstituted strain was designated AB1027. In addition, pWH1266-kan ${ }^{r}$-baeR was also introduced into the wild-type strain to generate the strain AB1028. Successful construction of the AB1027 and AB1028 strains was verified by RT-PCR. The expression of baeR was comparable in the wild-type and the baeR-reconstituted AB1027 strains, whereas baeR was overexpressed in AB1028 relative to the wild-type strain (data not shown).

\section{Minimal inhibitory concentration (MIC) determination}

To correlate BaeR with tigecycline susceptibility, the MIC of tigecycline was determined. For A. baumannii ATCC 17978, the MIC of tigecycline was $0.5 \mu \mathrm{g} / \mathrm{mL}$. However, the MIC of tigecycline for the baeR deletion mutant was $0.25 \mu \mathrm{g} / \mathrm{mL}$; baeR reconstitution restored the MIC to the wild-type level (MIC $0.5 \mu \mathrm{g} / \mathrm{mL}$ ). Moreover, the overexpression of baeR in AB1028 raised the MIC of tigecycline to $1 \mu \mathrm{g} / \mathrm{mL}$. The introduction of pWH1266 alone did not affect the MIC of tigecycline, whereas the MICs obtained with the induced tigecycline-resistant strain $\mathrm{ABtc}$ and the clinical tigecycline-resistant strain ABhl were 256 and $16 \mu \mathrm{g} / \mathrm{mL}$, respectively. These results indicate that BaeR is closely related to the tigecycline susceptibility of $A$. baumannii. 

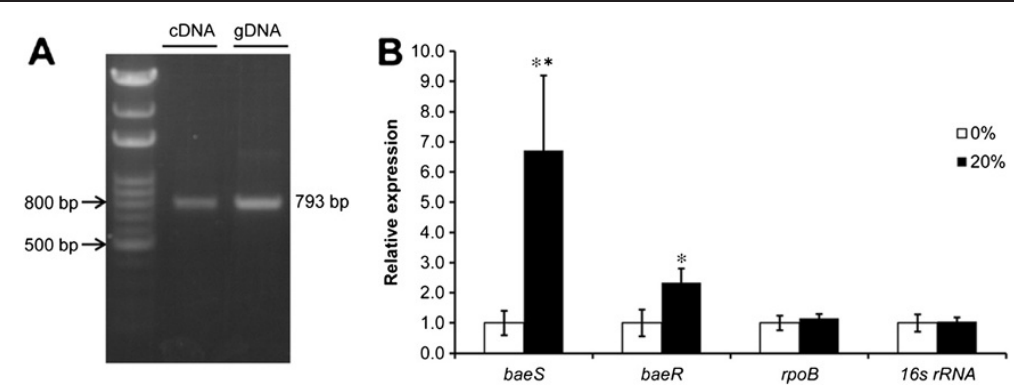

Figure 2 Evaluation of baeR and baeS expression. (A) The co-transcription of baeR and baes was determined by agarose gel electrophoresis of the product obtained by reverse transcription polymerase chain reaction (RT-PCR). Lane 2 (cDNA) and 3 (genomic DNA) reveal a 793-bp DNA fragment covering the junction between both the baeR and baes genes. (B) The relative transcript levels of baeR and baeS, as determined by RT-PCR, under different osmolarity conditions. The cells were grown on Luria-Bertani (LB) agar with or without $20 \%$ sucrose ( $\left.37^{\circ} \mathrm{C}, 220 \mathrm{rpm}\right)$. 165 rRNA and $r p o B$ genes were used as controls. The expression levels of baeR and baes were 2.3- and 6.7-fold higher in cells experiencing osmotic stress than those in cells grown without sucrose. The results are displayed as the means \pm SD from four independent experiments. $*, P<0.05 ; * *, P<0.01$.

Expression of the $a d e A B$ and baeSR genes in strains with different levels of tigecycline resistance

To further decipher the role of the BaeSR TCS and AdeAB in tigecycline resistance, we analyzed gene expression in the wild-type A. baumannii strain ATCC 17978 as well as the ABtc and ABhl1 strains. The quantitative realtime PCR (qRT-PCR) results showed that the expression levels of $a d e B$ were 216- and 53-fold higher in ABtc and $A B h 1$, respectively, than in the wild-type strain. ABtc and
ABhl1 also showed increased transcription of adeA1 and adeA2, although it was less marked than that of adeB (Figure 3A). The expression levels of baeS and baeR in ABtc increased 3.19 and 2.64-fold, respectively, compared with the wild-type strain, whereas those in ABhll only increased 1.93 and 1.39-fold, respectively (Figure 3B). Overall, the combination of the qRT-PCR results with the MIC assay above suggest that both BaeSR and AdeAB are involved in the tigecycline resistance of $A$. baumannii.

Table 2 Bacterial strains and plasmids used in this study

\begin{tabular}{|c|c|c|c|}
\hline \multicolumn{2}{|l|}{ Strain or plasmid } & \multirow{2}{*}{$\begin{array}{l}\text { Relevant feature(s) } \\
\text { Wild-type strain }\end{array}$} & \multirow{2}{*}{$\begin{array}{l}\text { Source or reference } \\
\text { ATCC }\end{array}$} \\
\hline A. baumannii strains & ATCC 17978 & & \\
\hline & AB1026 (AbaeR::kan') & $\begin{array}{l}\text { Derived from ATCC 17978. baeR mutant obtained by } \mathrm{kan}^{r} \\
\text { gene replacement }\end{array}$ & This study \\
\hline & AB1027 & AB1026 baeR::pWH1266 & This study \\
\hline & AB1028 & ATCC 17978 baeR::pWH1266 & This study \\
\hline & AB1029 & ATCC 17978 kan:: pWH1266 & This study \\
\hline & ABtc & Induced tigecycline resistant ATCC 17978 & This study \\
\hline & ABtcm $\left(\Delta b a e R:: k a n^{\prime}\right)$ & $\begin{array}{l}\text { Derived from ABtc. baeR mutant obtained by } \mathrm{kan}^{r} \text { gene } \\
\text { replacement }\end{array}$ & This study \\
\hline & ABhl1 & Tigecycline resistant clinical isolate & This study \\
\hline \multirow[t]{2}{*}{ E. coli strains } & XL1 blue & $\begin{array}{l}\text { recA1 endA1 gyrA96 thi-1 hsdR17 supE44 relA1 lac [F' proAB } \\
\left.\text { lacl }^{1} Z \Delta M 15 \operatorname{Tn} 10\left(\text { Tet }^{\prime}\right)\right]\end{array}$ & Stratagene \\
\hline & S17-1 (ATCC 47055) & thi pro hsdR hsdM recA[RP42-Tc::Mu- Km:::Tn7 (Tp'sm') Tra $\left.{ }^{+}\right]$ & ATCC \\
\hline \multirow[t]{7}{*}{ Plasmids } & pEX18Tc & Suicide vector containing $s a c B, T c^{r}$ & 40 \\
\hline & pSFS2A & Containing kan $^{r}$, an FRT site, FLP1, and CaSAT1 as a SAT1 flipper & 41 \\
\hline & pEX18Tc- $\Delta b a e:: k a n^{r}$ & $\begin{array}{l}\text { pEX } 18 T \text { T containing baeR upstream and downstream } \\
\text { fragments joined by a } k^{r}{ }^{r} \text { cassette }\end{array}$ & This study \\
\hline & pWH1266 (ATCC 77092) & E. coli-A. baumannii shuttle cloning vector, containing Ampr, Tet ${ }^{r}$ & 43 \\
\hline & $\mathrm{pC} 2 \mathrm{HP}$ & Provided $\mathrm{kan}^{r}$ for $\mathrm{pWH} 1266$ & 42 \\
\hline & pWH1266-kan ${ }^{r}$ & pWH1266 containing kan ${ }^{r}$ & This study \\
\hline & pWH1266-kan'-baeR & pWH1266-kan containing baeR & This study \\
\hline
\end{tabular}




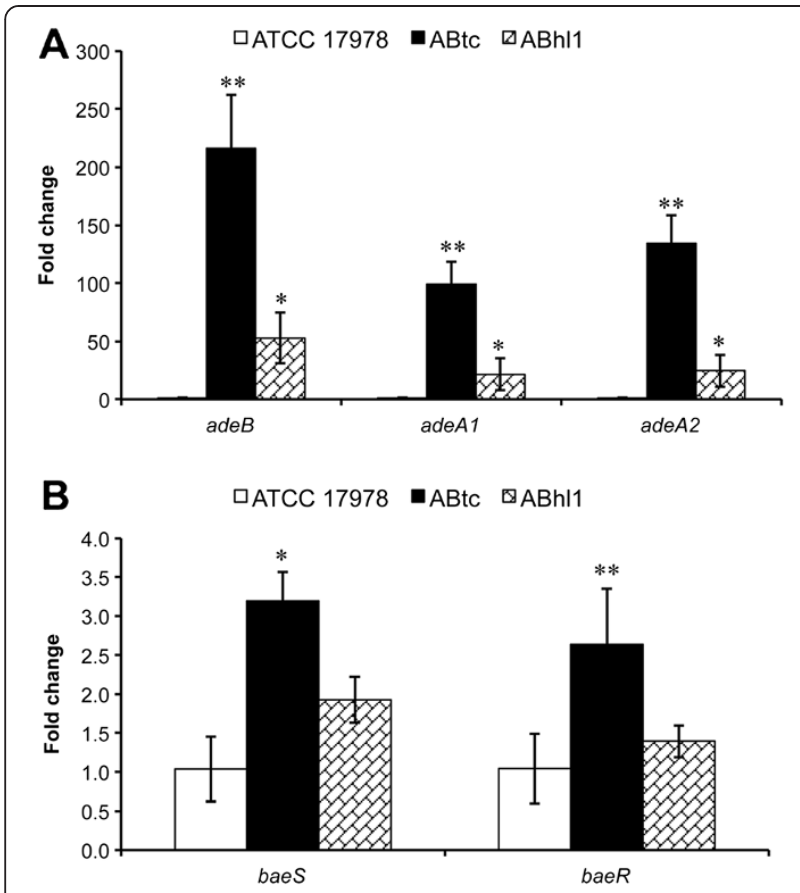

Figure 3 Transcript levels of the adeA, adeB, baeR, and baeS genes in $\boldsymbol{A}$. baumannii strains. ABtc and ABhl1 are laboratory-induced and clinically isolated tigecycline-resistant strains, respectively. The corresponding tigecycline minimum inhibitory concentrations (MICs) of ATCC 17978, ABtc, and ABhl1 were $0.5,256$, and $16 \mu \mathrm{g} / \mathrm{mL}$, respectively. Gene expression was detected by quantitative real-time PCR (qRT-PCR). (A) qRT-PCR showed that the expression levels of adeB in ABtc and ABhl1 were 216- and 53-fold higher than those in the wild-type strain, respectively. The adeA 1 expression levels in ABtc and ABhl1 were 99- and 22-fold higher than those in the wild-type strain, respectively, whereas the adeA2 expression levels in ABtc and ABhl1 were 134- and 25-fold higher. (B) The expression levels of baes and baeR in ABtc increased 3.19 and 2.64 times, respectively, compared with the wild-type strain, whereas those in ABhl1 only increased 1.93 and 1.39 times, respectively. 165 rRNA gene was used as a control. The results are displayed as the means \pm SD from four independent experiments. ${ }^{*}, P<0.05 ;{ }^{* *}, P<0.01$.

\section{Influence of the BaeSR TCS on adeAB efflux pump expression}

To understand whether $b a e R$ influenced the tigecycline MIC by affecting the $a d e A B$ efflux pump gene, the expression of adeA1, adeA2, and adeB in ATCC 17978, AB1026, AB1027, and AB1028 was analyzed by qRTPCR. The expression levels of adeB, adeA1, and adeA2 in AB1028 were approximately 2.9-, 2.1-, and 3-fold higher, respectively, than those in ATCC 17978, while the deletion of baeR from the wild-type strain decreased the expression levels of these three pump genes by $68.3 \%, 67.3 \%$, and $73.5 \%$, respectively (Figure 4A). The decreased expression of the pump genes can be partially restored by baeR reconstitution (Figure 4A). To determine the impact of baeR deletion on adeR expression, RT-PCR was also performed. No differences in adeR expression were observed between AB1026 and the wild- type strain (data not shown). Overall, these findings suggest that BaeR upregulates the expression of adeAB genes.

\section{Expression analysis of $a d e A B$ in induced tigecycline-}

resistant $A$. baumannii and its baeR mutant

To further confirm the role of baeR in the tigecycline resistance of $A$. baumannii via the AdeAB efflux pump, a baeR deletion mutant of ABtc (ABtcm) was constructed and $a d e A B$ expression was analyzed by qRT-PCR. The expression levels of adeB, adeA1, and adeA2 in ABtcm were $51.5,42.7 \%$, and $43.7 \%$ lower, respectively, than those in ABtc (Figure 4B). These data confirmed the contribution of BaeR to the regulation of AdeAB, which is essential to tigecycline resistance in A. baumannii.

\section{Time-kill assay}

To further compare the effects of BaeR on tigecycline susceptibility, time-kill assays were performed using ATCC 17978, AB1026, AB1027, and AB1028. There were no differences in the surviving colony forming units (CFUs) among these four strains when tigecycline was not added to the LB agar. In the presence of $0.25 \mu \mathrm{g} / \mathrm{mL}$ tigecycline, all tested strains had similar surviving CFU curves; the lowest value was observed at $4 \mathrm{~h}$, which was followed by regrowth (Figure 5A). Additionally, AB1026 showed a greater reduction in CFUs than the wild-type strain (e.g., 2.9- $\log _{10}$ versus $1.8-\log _{10}$ reduction, respectively, at $4 \mathrm{~h})$ throughout the assay period, which could be restored by $b a e R$ reconstitution. Increasing the tigecycline concentration to $0.5 \mu \mathrm{g} / \mathrm{mL}$ produced an even more marked 4.7$\log _{10}$ reduction in the CFUs of AB1026 at $8 \mathrm{~h}$, which was followed by regrowth. In contrast, a smaller reduction (2.1- $\log _{10}$ reduction at $8 \mathrm{~h}$ ) was observed for the wild-type strain (Figure $5 \mathrm{~B}$ ). However, baeR reconstitution did not fully restore the ability of AB1026 to resist $0.5 \mu \mathrm{g} / \mathrm{mL}$ tigecycline. AB1028 showed a slightly smaller reduction in CFUs than the wild-type strain in the presence of 0.25 and $0.5 \mu \mathrm{g} / \mathrm{mL}$ tigecycline. Therefore, the time-kill assay indicates that the BaeSR TCS plays a role in the tigecycline susceptibility of $A$. baumannii.

\section{Discussion}

Previous studies that investigated the regulation of AdeABC efflux pumps in A. baumannii primarily focused on the AdeRS TCS, which is located upstream of the adeABC operon and is transcribed in the opposite direction [15]. Several point mutations in adeR or adeS have been proposed as the major cause of AdeABC efflux pump overexpression, including a threonine-to-methionine substitution at position 153 [15], a glycine-to-aspartate mutation at position 30 [24], an alanine-to-valine substitution at position 94 of AdeS [25], or a proline-to-leucine substitution at position 116 of AdeR [15]. However, the effect of 

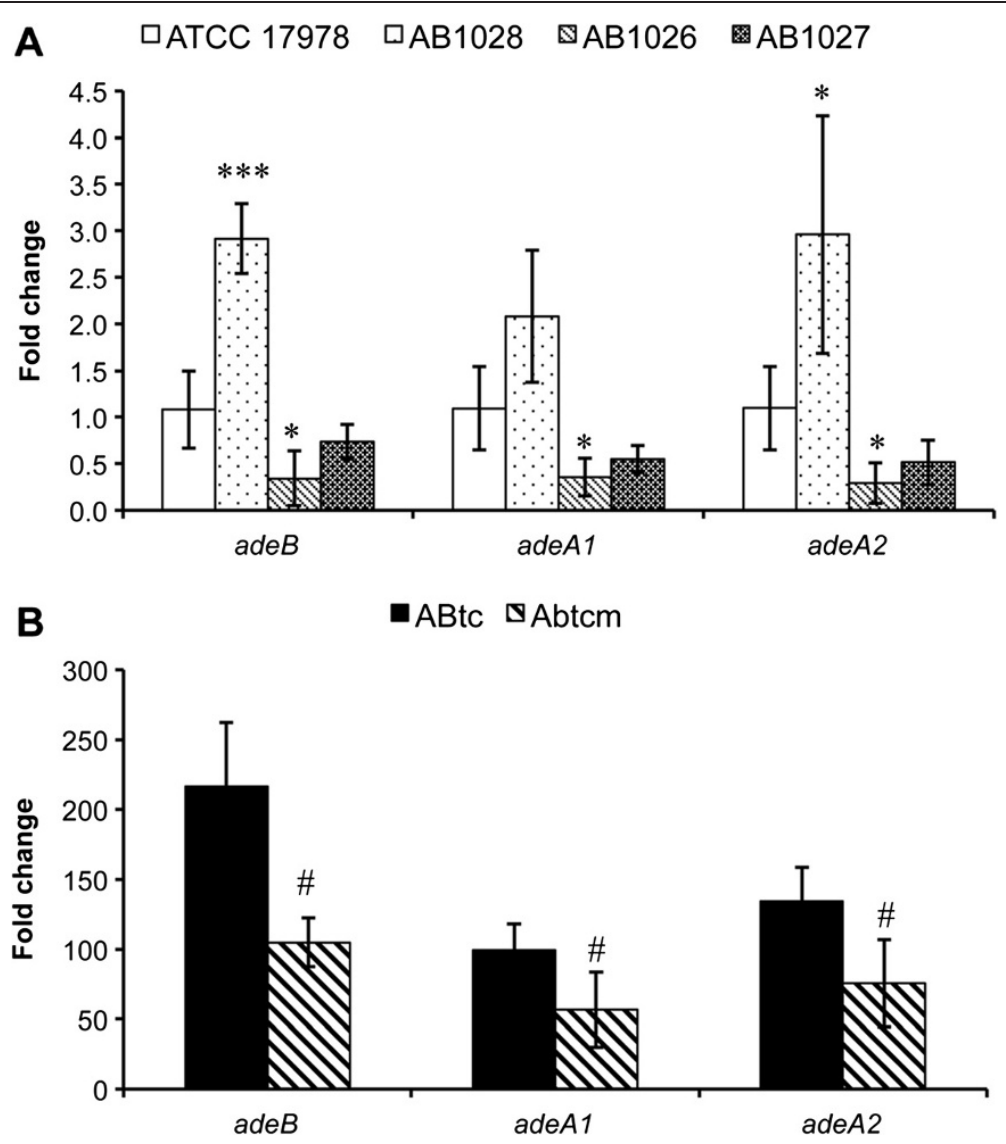

Figure 4 Transcript levels of the adeA and adeB genes in different strains of $\boldsymbol{A}$. baumannii. AB1026, AB1027, and AB1028 are the baeR deletion mutant, baeR reconstitution, and wild-type with baeR overexpression strains, respectively. ABTcm is the bae $R$ deletion mutant of $A B t c$, which was a laboratory-induced tigecycline-resistant strain. The relative expression of adeB, adeA1, and adeA2 was determined by qRT-PCR.

(A) The expression levels of adeB, adeA1, and adeA2 in AB1028 were approximately 2.9-, 2.1-, and 3-fold higher, respectively, than those in ATCC 17978 , while the deletion of baeR in the wild-type strain decreased the expression levels of these three pump genes by $68.3 \%, 67.3 \%$, and $73.5 \%$, respectively. The decreased expression of the pump genes can be partially restored by baeR reconstitution. (B) The expression levels of adeB, adeA1, and adeA2 in ABtcm were 51.5\%, 42.7\%, and 43.7\% lower, respectively, than those in ABtc. 16S rRNA gene was used as a control. The results are displayed as the means $\pm \mathrm{SD}$ from three independent experiments. ${ }^{*}, P<0.05 ;{ }^{* *}, P<0.001$. $\#, P<0.05$ between ABtc and ABtcm.

AdeR or AdeS mutations on the expression of AdeABC is not always consistent. Different tigecycline MICs were observed in two transformed strains with the same mutations in the DNA-binding domain of the AdeR protein [16]. ade $A B C$-overexpressing mutants that did not carry any mutations in adeRS compared with their isogenic parents were also reported $[7,25]$. Another mechanism leading to the overexpression of AdeABC involves the transposition of an ISAba1 copy into adeS [15], which stimulates AdeR to interact with and activate the adeABC promoter [16]. In contrast to the results of the abovementioned studies of AdeRS, four imipenem-resistant $A$. baumannii strains carrying adeB but lacking adeRS were identified by Hou et al. [26], suggesting that another regulatory mechanism may be involved. Henry et al. reported that BaeSR was associated with the increased expression of the multidrug resistance-associated efflux pump genes macAB-tolC and adeIJK in their transcriptional analysis of lipopolysaccharide-deficient A. baumannii 19606R [27]. Therefore, the role of BaeSR in the expression of the AdeABC efflux pump deserves investigation. Our data demonstrate that BaeSR influences the tigecycline susceptibility of A. baumannii ATCC 17978 through its positive regulation of the transcription of transporter genes adeA and $a d e B$. This result supported the possibility that other TCSs aside from AdeRS may be involved in the regulation of the AdeABC efflux pump in A. baumannii.

Most $A$. baumannii strains have an RND efflux pump, AdeABC, which has a three-component structure with AdeB forming the transmembrane component, AdeA forming the inner membrane fusion protein, and AdeC forming the outer membrane protein [9]. However, according to the NCBI GenBank database, A. baumannii ATCC 17978 lacks an ade $C$ gene but has two adeA genes and one adeB gene. A. baumannii AYE, A. baumannii ACICU, $A$. baumannii ATCC 19606, and A. baumannii TYTH-1 all 

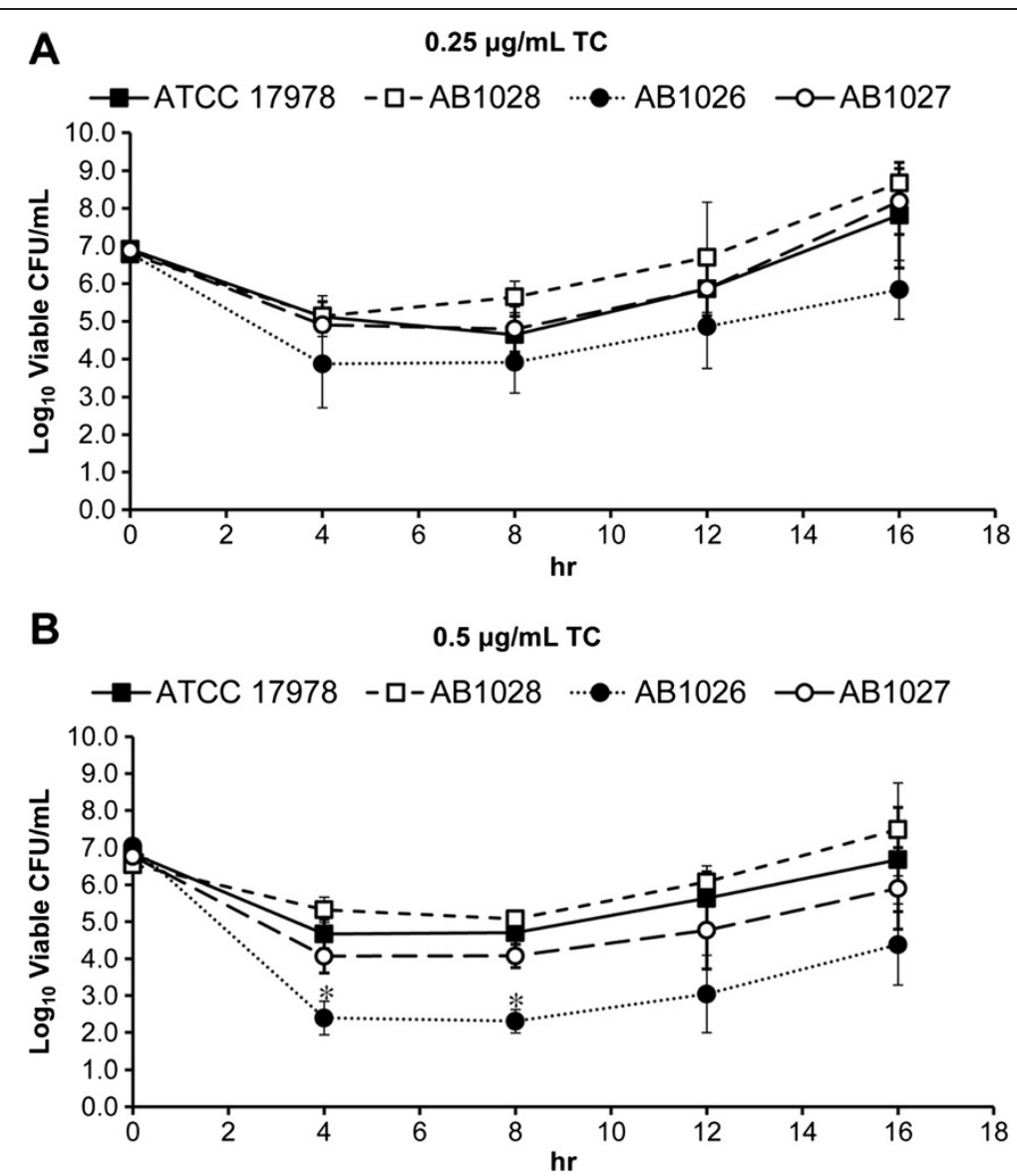

Figure 5 Time-kill assays for ATCC 17978, AB1026, AB1027, and AB1028 with $0.25 \mu \mathrm{g} / \mathrm{mL}$ (A) and $0.5 \mu \mathrm{g} / \mathrm{mL}$ (B) tigecycline. In the presence of $0.25 \mu \mathrm{g} / \mathrm{mL}$ tigecycline, all tested strains showed similar surviving colony forming unit (CFU) curves, in which the lowest value occurred at $4 \mathrm{~h}$ and was followed by regrowth. AB1026 had a greater CFU reduction than the wild-type strain throughout the assay period, which could be restored by baeR reconstitution. Increasing the tigecycline concentration to $0.5 \mu \mathrm{g} / \mathrm{mL}$ resulted in a marked $4.7-\log _{10}$ CFU reduction for AB1026 at 8 h, which was followed by regrowth, whereas a smaller reduction was observed for the wild-type strain. baeR reconstitution did not fully restore the ability of AB1026 to resist $0.5 \mu \mathrm{g} / \mathrm{mL}$ tigecycline. AB1028 had a slight, though not significant, CFU reduction compared to the wild-type strain in the presence of 0.25 or $0.5 \mu \mathrm{g} / \mathrm{mL}$ tigecycline. Viable counts represented by CFUs were determined at time 0 and at $4,8,12$, and $16 \mathrm{~h}$ after inoculation. A time-kill curve was constructed for each strain. The results are displayed as the means \pm SD from three independent experiments. ${ }^{*}, P<0.05$.

possess an AdeC-like outer membrane protein. Marchand et al. constructed a clinical $A$. baumannii strain with an inactivated $a d e C$. This derivative mutant displayed resistance to the various substrates of the AdeABC pump that was similar to that of the wild-type strain, indicating that adeC is not essential for resistance [15]. Because $a d e C$ was not found in $41 \%$ of the clinical isolates carrying adeRSade $A B$ in one study [28], it is reasonable to deduce that AdeAB could recruit another outer membrane protein to form a functional tripartite complex [29].

The first description of tigecycline non-susceptibility was reported by Peleg et al. [7]. These authors found that the efflux pump inhibitor phenyl-arginine- $\beta$-naphthylamide could cause a four-fold reduction in the MIC of tigecycline in two tigecycline-non-susceptible isolates. The qRT-PCR results showed 40-fold and 54-fold increases in
adeB expression in these two isolates compared to that observed in a tigecycline-susceptible isolate. Their finding is consistent with our comparison of tigecycline MICs and expression levels of AdeAB among the wild-type, ABhl1, and ABtc strains. Despite the important role of AdeABC in antibiotic resistance, this efflux pump operon is cryptic in natural isolates of A. baumannii [15,30]. Antibiotic exposure, including exposure to tigecycline, could induce pump overexpression, resulting in drug resistance [29]; this was observed in our ABtc strain. Furthermore, there was a statistically significant linear relationship between log-transformed adeA expression values and log-transformed MICs of tigecycline in clinical isolates of the A. calcoaceticus-A. baumannii complex, indicating that the overexpression of the AdeABC efflux pump is a prevalent mechanism for this resistance phenotype [31]. 
The modest increase in AdeAB pump gene expression in AB1028 relative to the wild-type strain may have been due to the overexpression of BaeSR. However, because ABtcm had only moderately reduced $a d e B$, adeA1, and adeA2 expression levels relative to ABtc, we proposed that control mechanisms aside from BaeSR, such as sequence changes in $a d e R$ or adeS, were responsible for the overexpression of these pump genes. The regulators that are involved in efflux gene expression are either local or global regulators [32]. One of the most well-studied examples is the AcrAB-TolC system of E. coli [33]. This system is under the control of the local repressor gene $a c r R$, which negatively regulates the transcription of $\operatorname{acr} A B$. On the other hand, global stress conditions are assumed to result in the generation of global transcription regulators. These regulators are unlikely to be MarA, SoxS, or Rob, but could be their homologs. Such regulators increase the transcription of not only $a c r A B$ but also $a c r R$, which functions as a secondary modulator to repress $\operatorname{acr} A B$. Fernando et al. demonstrated that the transcription patterns of both $a d e B$ and adeJ are cell density-dependent and similar, indicating a role for global regulatory mechanisms in the expression of these genes in A. baumannii [34]. Two-component regulatory systems mediate the adaptive responses of bacterial cells to a broad range of environmental stimuli [35]. In this study, qRT-PCR analysis of baeSR expression under high sucrose conditions suggested that this TCS was involved in the regulation related to this stress condition. Therefore, we propose that BaeSR, which functions as an envelope stress response system to external stimuli, also influences the transcription of $a d e A B$ in $A$. baumannii by functioning as a regulator of global transcription. Meanwhile, the well-described $a d e R$ is an example of a local regulator that activates adeABC expression $[15,16]$. However, the relationship between BaeSR and AdeRS must be further clarified. Because the expression of adeRS was only marginally increased in the baeSR deletion mutants in this study, we assume that the crosstalk between these TCSs might be absent or only very weak. The question of whether other TCSs are involved in the regulation of the AdeABC efflux pump and how they interact in $A$. baumannii merits further investigation.

\section{Conclusions}

In this study, we showed for the first time that the BaeSR TCS influences the tigecycline susceptibility of $A$. baumannii by positively regulating the RND efflux pump genes ade $A$ and $a d e B$. However, whether BaeSR can also contribute to tigecycline resistance through other transporter genes, such as macAB-tolC and adeIJK, is not yet clear, and related studies are underway. Overall, this finding highlights the complexity of AdeABC transporter regulation and could be a starting point for understanding the role of TCSs in the antimicrobial susceptibility of bacteria.

\section{Methods}

Bacterial strains, plasmids, growth conditions, and antibiotic susceptibility testing

The bacterial strains and plasmids used in this study are listed in Table 2 . The cells were grown at $37^{\circ} \mathrm{C}$ in LB broth and agar. To determine the MIC, a broth microdilution method was used according to the 2012 CLSI guidelines [36]. Briefly, bacteria were inoculated into $1 \mathrm{~mL}$ cationadjusted Mueller-Hinton broth (CAMHB) (Sigma-Aldrich, St. Louis, MO) containing different concentrations of tigecycline (Pfizer, Collegeville, PA) to reach $\approx 5 \times 10^{5} \mathrm{CFU} / \mathrm{mL}$, and the cultures were incubated at $37^{\circ} \mathrm{C}$ for $24 \mathrm{~h}$. The lowest tigecycline concentration that completely inhibited bacterial growth was defined as the MIC, and growth was determined by unaided eyes and by measuring optical densities (ODs) using a spectrophotometer. On the basis of the report published by Pachón-Ibáñez et al., the provisional MIC breakpoints for tigecycline are $\leq 2,4$, and $\geq 8 \mu \mathrm{g} / \mathrm{mL}$ to designate susceptible, intermediate, and resistant strains, respectively [37].

\section{DNA manipulation}

Plasmid DNA was prepared with the FavorPrep ${ }^{\mathrm{Tm}}$ Plasmid DNA Extraction Mini Kit (Favorgen, Ping-Tung, Taiwan). A. baumannii genomic DNA was extracted as described previously [38]. PCR amplification of the DNA was performed in a Thermo Hybaid PXE 0.2 HBPX02 Thermal Cycler (Thermo Scientific, Redwood, CA), using ProTa ${ }^{\mathrm{Tm}}$ DNA Polymerase (Protech, Taipei, Taiwan) or the KAPA $\mathrm{HiFi}^{\mathrm{im}}$ PCR Kit (Kapa Biosystems, Boston, MA). DNA fragments were extracted from agarose gels and purified using the GeneKlean Gel Recovery \& PCR CleanUp Kit (MDBio, Inc., Taipei, Taiwan). Nucleotide sequences of the PCR products were verified using an ABI 3730XL DNA Analyzer (Applied Biosystems, South San Francisco, CA).

\section{RNA isolation, RT-PCR, and qRT-PCR}

For total RNA isolation, A. baumannii ATCC 17978 was grown overnight in $\mathrm{LB}$ broth $\left(37^{\circ} \mathrm{C}, 220 \mathrm{rpm}, 16 \mathrm{~h}\right)$ to reach an $\mathrm{OD}_{600}$ of approximately 6.5 . The overnight cultures were sub-cultured at a 1:100 dilution in $25 \mathrm{~mL}$ fresh LB medium. The cells were grown to mid-log phase and harvested by centrifugation at $4^{\circ} \mathrm{C}$. The cell pellets were resuspended in $200 \mu \mathrm{L}$ ice-cold RNA extraction buffer (0.1 M Tris-Cl [pH 7.5], 0.1 M LiCl, 0.01 M ethylenediaminetetraacetic acid [pH 8.0], 5\% sodium dodecyl sulfate [SDS], $2 \% \beta$-mercaptoethanol), and $200 \mu \mathrm{L}$ ice-cold phenol-chloroform-isoamyl alcohol (PCIA [25:24:1], $\mathrm{pH} 4.5$ ) was added and vortexed for $2 \mathrm{~min}$. The supernatants were then collected by centrifugation, added to $200 \mu \mathrm{L}$ ice-cold PCIA, and mixed well. This step was repeated 
three times. Then, RNA was precipitated with ethanol at $-80^{\circ} \mathrm{C}$ overnight and collected by centrifugation at maximum speed for $5 \mathrm{~min}$. The RNA pellets were dissolved in 25-100 $\mu \mathrm{L}$ diethylpyrocarbonate-treated water. DNA was removed using Ambion ${ }^{\circ}$ TURBO $^{\text {ma }}$ DNase (Life Technologies, Grand Island, NY), and cDNA was synthesized by reverse transcription using High-Capacity cDNA Reverse Transcriptase Kits (Applied Biosystems). The cDNAs were used in PCR reactions with different primers (Table 1).

qRT-PCR was carried out with a StepOne ${ }^{\text {ts }}$ Real-Time PCR System (Life Technologies). The primers used for qRT-PCR are listed in Table 1 . Briefly, each $20-\mu \mathrm{L}$ reaction mixture contained $25 \mathrm{ng}$ cDNA, $10 \mu \mathrm{L}$ Power SYBR green PCR master mix (Life Technologies), and $300 \mathrm{nM}$ each forward and reverse primer. The reactions were performed with 1 cycle at $95^{\circ} \mathrm{C}$ for $10 \mathrm{~min}$ followed by 40 cycles of $95^{\circ} \mathrm{C}$ for $15 \mathrm{~s}$ and $60^{\circ} \mathrm{C}$ for $1 \mathrm{~min}$. The $16 \mathrm{~S}$ rRNA transcript was used as an endogenous control for the qRT-PCR. The data were analyzed using StepOne v2.1 software (Life Technologies).

\section{Induction of tigecycline resistance}

To induce tigecycline resistance, serial passaging was performed as previously described [39] with some modifications. Briefly, on day 1, $3 \mathrm{~mL}$ of LB broth containing tigecycline at the MIC was inoculated with A. baumannii (passage 1), and the cultures were incubated at $37^{\circ} \mathrm{C}$ with shaking $(220 \mathrm{rpm})$. On day $3,30 \mu \mathrm{L}$ of the culture was transferred to $3 \mathrm{~mL}$ of LB broth containing tigecycline at $8 \times$ the MIC (passage 2), and the cultures were again incubated at $37^{\circ} \mathrm{C}$ with shaking $(220 \mathrm{rpm})$. On day $5,30 \mu \mathrm{L}$ of the culture was transferred into LB broth containing tigecycline at $16 \times$ the MIC (passage 3), and the cultures were again incubated at $37^{\circ} \mathrm{C}$ with shaking $(220 \mathrm{rpm})$. This passaging was repeated on day 7 (passage 4). On day 9 , aliquots $(3 \mathrm{~mL})$ of the cultures were mixed with $10 \%$ glycerol and stored at $-80^{\circ} \mathrm{C}$ until use. Daily passaging in tigecycline-free LB was conducted for 30 days for both ATCC 17978 and the clinical strain.

\section{Construction of baeR deletion mutants and baeR reconstituted strains}

To assess the contribution of BaeR to the regulation of tigecycline resistance, baeR deletion mutants of A. baumannii ATCC 17978 were constructed as previously described [23] with some modifications. The suicide vector pEX18Tc [40] was first cloned with a 953-bp DNA fragment carrying a kanamycin resistance cassette, which was PCR-amplified from the pSFS2A plasmid [41], to generate pEX18Tc-kan ${ }^{r}$. DNA fragments carrying the upstream and downstream regions of the baeR gene, referred to as baeR-up and baeR-dw, were independently amplified by PCR using the primer pairs baeR-up-SalI-F and baeR-up-BamHI-R or baeR-dw-KpnI-F and baeR$\mathrm{dw}$-SacI-R (Table 1). The baeR-up fragment (1,119 bp) was digested with SalI and BamHI enzymes, whereas the baeR-dw fragment (1,120 bp) was digested with KpnI and SacI enzymes (Additional file 4: Figure S4A). Both enzyme-digested DNA fragments were then independently cloned into the corresponding restriction sites of pEX18Tc-kan ${ }^{r}$, generating pEX18Tc-kan ${ }^{r}$-baeR-flanking. The resultant plasmid was then transformed into the $E$. coli S17-1 strain using the standard $\mathrm{CaCl}_{2} /$ heat shock method [38]. Then, trans-conjugation was performed between E. coli S17-1 donor cells and A. baumannii ATCC 17978 recipient cells to transfer and integrate pEX18Tc$\mathrm{kan}^{r}$-baeR-flanking into the chromosome of ATCC 17978 (Additional file 4: Figure S4B). By growing the ATCC 17978 conjugate cells on LB agar containing $10 \%$ sucrose, the cells were able to resolve the suicide plasmid pEX18Tc (Additional file 4: Figure S4C). Sucrose-resistant colonies were examined to verify that they had the kanamycinresistant phenotype as a result of plasmid eviction. The absence of the baeR gene sequence in the genome was verified by PCR and RT-PCR and further confirmed by Southern blot hybridization.

To reconstitute the baeR gene in the baeR-deleted mutants, a DNA fragment carrying the entire baeR gene sequence was generated by PCR using the genomic DNA of A. baumannii ATCC 17978 as the template. Briefly, a kanamycin resistance cassette was first amplified from the pC2HP vector [42] and cloned into the E. coli/Acinetobacter shuttle vector pWH1266 [43,44] (Additional file 5: Figure S5A and S5B). Subsequently,

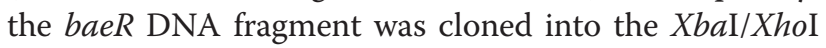
restriction sites (Additional file 5: Figure S5C). The plasmid was transformed into the wild-type strain and the baeR deletion mutants by electroporation, thus creating the baeR-overexpressing strain and complemented mutant strains, respectively. The overexpression and baeR-reconstituted strains were selected on LB agar containing $10 \mu \mathrm{g} / \mathrm{mL}$ tetracycline and were further verified by PCR (Additional file 5: Figure S5D) and RT-PCR (Additional file 2: Figure S2).

\section{Southern blot hybridization}

Southern blot analysis was performed as reported in a previous publication [45]. Genomic DNA was extracted, and approximately $10 \mu \mathrm{g}$ was digested with $B c l$ I overnight at $50^{\circ} \mathrm{C}$. The DNA was then separated on a $0.8 \%$ agarose gel containing 1:10,000 SYBR Safe gel stain (Invitrogen, Grand Island, NY), transferred onto a positively charged nylon membrane (Pall Corporation, Port Washington, NY) via the alkaline transfer method [38], and fixed by baking at $80^{\circ} \mathrm{C}$ for $2 \mathrm{~h}$. The membrane was hybridized with an $\left[\alpha-{ }^{32} \mathrm{P}\right] \mathrm{dCTP}$-labeled baeS probe (Additional file 3: Figure S3A) using prehybridization 
buffer (6x saline sodium citrate [SSC; $1 \times$ SSC is $0.15 \mathrm{M}$ $\mathrm{NaCl}$ plus $0.015 \mathrm{M}$ sodium citrate], $5 \times$ Denhardt's reagent, $0.5 \%$ SDS, $100 \mu \mathrm{g} / \mathrm{mL}$ salmon sperm DNA, and $50 \%$ formamide) at $42^{\circ} \mathrm{C}$ overnight. The membrane was then washed and visualized by autoradiography.

\section{Time-kill assay}

The time-kill assays were carried out in duplicate as previously described [46] with some modifications. Briefly, cells were grown to $\log$ phase and sub-cultured into $10 \mathrm{~mL}$ CAMHB broth without (control) or with tigecycline $(0.25$ or $0.5 \mu \mathrm{g} / \mathrm{mL})$ to a cell density of approximately $5 \times 10^{5} \mathrm{CFU} / \mathrm{mL}$. The cultures were incubated in an ambient atmosphere at $37^{\circ} \mathrm{C}$. At different time points $(0,4,8,12$, and $16 \mathrm{~h})$ after inoculation, $0.1 \mathrm{~mL}$ of the culture was removed from each tube and 10-fold serially diluted. Then, $25 \mu \mathrm{L}$ of each diluted cell suspension was spotted onto LB agar in duplicate. Viable cell counts were determined, the duplicates were averaged, and the data were plotted.

\section{Additional files}

Additional file 1: Figure S1. Verification of the baeR deletion mutants (A) Diagram of the baeR gene and deletion mutant verification using appropriate primers. (B) Successful baeR gene fragment deletion was deduced based on a change in the PCR band size from $4539 \mathrm{bp}$ to 4884 bp.

Additional file 2: Figure S2. Southern blot analysis. (A) Genomic DNA from the baeR deletion mutant and the parental strain was digested by $B C l l$. The location of the specific DNA probe is shown. (B) The bands corresponding to 6.7-kb and 2.8-kb fragments are indicated. Four independent clones of AB1026 are included.

Additional file 3: Figure S3. Construction of the baeR deletion mutant. (A) A single crossover between pEX18Tc containing baeR upstream and downstream sequences joined by a $\mathrm{kan}^{r}$ cassette and the ATCC 17978 chromosome. (B) Two mechanisms by which the plasmid can integrate into the chromosome are diagrammed. (C) The suicide plasmid was excised by $10 \%$ sucrose counter-selection and selection of the in-frame baeR deletion strain with kanamycin.

Additional file 4: Figure S4. Shuttle vector pWH1266 and verification of pWH1266 introduction into different strains of Acinetobacter baumannii. (A) pWH1266. (B) pWH1266 with kanamycin cassette insertion. (C) baeR insertion into the Xbal/Xhol restriction sites in pWH1266. (D) Successful baeR gene fragment insertion into the kanamycin cassette was deduced based on a change in the PCR band size from 1375 bp to 983 bp. AB1027, AB1028, and AB1029 represent the baeR reconstituted strain, the baeR-overexpressing strain, and the $A$. baumannii ATCC 17978 strain with pWH1266, respectively.

Additional file 5: Figure S5. baeR gene expression in different $A$. baumannii strains as determined by reverse transcription polymerase chain reaction. No baeR expression could be observed in AB1026. AB1027 was the baeR-reconstituted strain derived from AB1026, which had a baeR expression level similar to that of the wild-type strain. AB1028 and AB1029 represent the baeR-overexpressing strain and A. baumannii ATCC 17978 with pWH1266, respectively.

\section{Competing interests}

The authors declare that they have no competing interests.

\section{Authors' contributions}

MFL conceived the study design and drafted the manuscript. YYL performed the laboratory work, including the mutant construction and complementation, gene expression, and time-kill assays. HWL carried out the MIC determinations. CYL participated in the overall design of this study and assisted in writing the manuscript. All authors have read and approved the final manuscript.

\section{Acknowledgements}

This study was supported by a grant from the National Taiwan University Hospital, Chu-Tung Branch. The authors also thank Dr. Kia-Chih Chang (Tzu Chi University, Taiwan) for providing the clinical A. baumannii strains and Dr. Ming-Li Liou (Yuanpei University, Taiwan) for providing the wild-type strain. We also thank Jeng-Yi Chen for his technical assistance.

\section{Author details}

1Department of Medicine, National Taiwan University Hospital Chu-Tung Branch, Hsin-Chu County, Taiwan. ${ }^{2}$ Institute of Molecular and Cellular Biology, National Tsing Hua University, Hsin-Chu City, Taiwan. ${ }^{3}$ Laboratory Medicine Division, National Taiwan University Hospital Hsin-Chu Branch, Hsin-Chu City, Taiwan. ${ }^{4}$ Department of Life Science, National Tsing Hua University, Hsin-Chu City, Taiwan.

Received: 28 December 2013 Accepted: 25 April 2014

Published: 9 May 2014

\section{References}

1. Fournier PE, Richet $\mathrm{H}$ : The epidemiology and control of Acinetobacter baumannii in health care facilities. Clin Infect Dis 2006, 42(5):692-699.

2. Perez F, Hujer AM, Hujer KM, Decker BK, Rather PN, Bonomo RA: Global challenge of multidrug-resistant Acinetobacter baumannii. Antimicrob Agents Chemother 2007, 51(10):3471-3484.

3. Mendes RE, Farrell DJ, Sader HS, Jones RN: Comprehensive assessment of tigecycline activity tested against a worldwide collection of Acinetobacter spp. (2005-2009). Diagn Microbiol Infect Dis 2010, 68(3):307-311

4. Lauderdale TL, Clifford McDonald L, Shiau YR, Chen PC, Wang HY, Lai JF, Ho M: The status of antimicrobial resistance in Taiwan among gram-negative pathogens: the Taiwan surveillance of antimicrobial resistance (TSAR) program, 2000. Diagn Microbiol Infect Dis 2004, 48(3):211-219.

5. Gordon NC, Wareham DW: Multidrug-resistant Acinetobacter baumannii: mechanisms of virulence and resistance. Int J Antimicrob Agents 2010, 35(3):219-226.

6. Rose WE, Rybak MJ: Tigecycline: first of a new class of antimicrobial agents. Pharmacotherapy 2006, 26(8):1099-1110.

7. Peleg AY, Adams J, Paterson DL: Tigecycline Efflux as a Mechanism for Nonsusceptibility in Acinetobacter baumannii. Antimicrob Agents Chemother 2007, 51(6):2065-2069.

8. Ruzin A, Keeney D, Bradford PA: AdeABC multidrug efflux pump is associated with decreased susceptibility to tigecycline in Acinetobacter calcoaceticus-Acinetobacter baumannii complex. J Antimicrob Chemother 2007, 59(5):1001-1004

9. Vila J, Marti S, Sanchez-Cespedes J: Porins, efflux pumps and multidrug resistance in Acinetobacter baumannii. J Antimicrob Chemother 2007, 59(6):1210-1215.

10. Alm E, Huang K, Arkin A: The evolution of two-component systems in bacteria reveals different strategies for niche adaptation. PLoS Comput Biol 2006, 2(11):e143.

11. West $A H$, Stock $A M$ : Histidine kinases and response regulator proteins in two-component signaling systems. Trends Biochem Sci 2001, 26(6):369-376.

12. Sun S, Negrea A, Rhen M, Andersson Dl: Genetic analysis of colistin resistance in Salmonella enterica serovar Typhimurium. Antimicrob Agents Chemother 2009, 53(6):2298-2305.

13. Kishii $\mathrm{R}$, Takei M: Relationship between the expression of ompF and quinolone resistance in Escherichia coli. J Infect Chemother 2009, 15(6):361-366

14. Barrow K, Kwon DH: Alterations in two-component regulatory systems of phoPQ and pmrAB are associated with polymyxin $B$ resistance in clinical isolates of Pseudomonas aeruginosa. Antimicrob Agents Chemother 2009, 53(12):5150-5154. 
15. Marchand I, Damier-Piolle L, Courvalin P, Lambert T: Expression of the RND-type efflux pump AdeABC in Acinetobacter baumannii is regulated by the AdeRS two-component system. Antimicrob Agents Chemother 2004, 48(9):3298-3304.

16. Sun JR, Perng CL, Chan MC, Morita Y, Lin JC, Su CM, Wang WY, Chang TY, Chiveh TS: A truncated AdeS kinase protein generated by ISAba1 insertion correlates with tigecycline resistance in Acinetobacter baumannii. PLOS ONE 2012, 7(11):e49534.

17. Bury-Mone S, Nomane $Y$, Reymond N, Barbet R, Jacquet E, Imbeaud S, Jacq A, Bouloc P: Global analysis of extracytoplasmic stress signaling in Escherichia coli. PLoS Genet 2009, 5(9):e1000651.

18. Leblanc SK, Oates CW, Raivio TL: Characterization of the induction and cellular role of the BaeSR two-component envelope stress response of Escherichia coli. J Bacteriol 2011, 193(13):3367-3375.

19. Appia-Ayme C, Patrick E, Sullivan MJ, Alston MJ, Field SJ, AbuOun M, Anjum MF, Rowley G: Novel inducers of the envelope stress response BaeSR in Salmonella Typhimurium: BaeR is critically required for tungstate waste disposal. PLOS ONE 2011, 6(8):e23713.

20. Rosner JL, Martin RG: Reduction of cellular stress by TolC-dependent efflux pumps in Escherichia coli indicated by BaeSR and CpxARP activation of spy in efflux mutants. J Bacterio/ 2013, 195(5):1042-1050.

21. Nishino K, Honda T, Yamaguchi A: Genome-wide analyses of Escherichia coli gene expression responsive to the BaeSR two-component regulatory system. J Bacteriol 2005, 187(5):1763-1772.

22. Baranova N, Nikaido H: The BaeSR Two-Component Regulatory System Activates Transcription of the yegMNOB ( $m d t A B C D$ ) Transporter Gene Cluster in Escherichia coli and Increases Its Resistance to Novobiocin and Deoxycholate. J Bacteriol 2002, 184(15):4168-4176.

23. Sugawara $\mathrm{E}$, Nikaido $\mathrm{H}$ : OmpA is the principal nonspecific slow porin of Acinetobacter baumannii. J Bacteriol 2012, 194(15):4089-4096.

24. Coyne S, Guigon G, Courvalin P, Perichon B: Screening and quantification of the expression of antibiotic resistance genes in Acinetobacter baumannii with a microarray. Antimicrob Agents Chemother 2010, 54(1):333-340

25. Hornsey M, Ellington MJ, Doumith M, Thomas CP, Gordon NC, Wareham DW, Quinn J, Lolans K, Livermore DM, Woodford N: AdeABC-mediated efflux and tigecycline MICs for epidemic clones of Acinetobacter baumannii. J Antimicrob Chemother 2010, 65(8):1589-1593.

26. Hou PF, Chen XY, Yan GF, Wang YP, Ying CM: Study of the correlation of imipenem resistance with efflux pumps AdeABC, AdelJK, AdeDE and AbeM in clinical isolates of Acinetobacter baumannii. Chemotherapy 2012, 58(2):152-158

27. Henry R, Vithanage N, Harrison P, Seemann T, Coutts S, Moffatt JH, Nation RL, Li J, Harper M, Adler B, Boyce JD: Colistin-resistant, lipopolysaccharide-deficient Acinetobacter baumannii responds to lipopolysaccharide loss through increased expression of genes involved in the synthesis and transport of lipoproteins, phospholipids, and poly-beta-1,6-N-acetylglucosamine. Antimicrob Agents Chemother 2012, 56(1):59-69.

28. Nemec A, Maixnerova M, van der Reijden TJ, van den Broek PJ, Dijkshoorn L: Relationship between the AdeABC efflux system gene content, netilmicin susceptibility and multidrug resistance in a genotypically diverse collection of Acinetobacter baumannii strains. J Antimicrob Chemother 2007, 60(3):483-489

29. Coyne $S$, Courvalin P, Perichon B: Efflux-mediated antibiotic resistance in Acinetobacter spp. Antimicrob Agents Chemother 2011, 55(3):947-953.

30. Magnet S, Courvalin P, Lambert T: Resistance-nodulation-cell division-type efflux pump involved in aminoglycoside resistance in Acinetobacter baumannii strain BM4454. Antimicrob Agents Chemother 2001, 45(12):3375-3380.

31. Ruzin A, Immermann FW, Bradford PA: RT-PCR and statistical analyses of adeABC expression in clinical isolates of Acinetobacter calcoaceticusAcinetobacter baumannii complex. Microb Drug Resist 2010, 16(2):87-89.

32. Wieczorek P, Sacha P, Hauschild T, Zorawski M, Krawczyk M, Tryniszewska E: Multidrug resistant Acinetobacter baumannii-the role of AdeABC (RND family) efflux pump in resistance to antibiotics. Folia Histochem Cytobiol 2008, 46(3):257-267.

33. Ma D, Alberti M, Lynch C, Nikaido H, Hearst JE: The local repressor AcrR plays a modulating role in the regulation of $a c r A B$ genes of Escherichia coli by global stress signals. Mol Microbiol 1996, 19(1):101-112.
34. Fernando D, Kumar A: Growth phase-dependent expression of RND efflux pump- and outer membrane porin-encoding genes in Acinetobacter baumannii ATCC 19606. J Antimicrob Chemother 2012, 67(3):569-572.

35. Stock AM, Robinson VL, Goudreau PN: Two-component signal transduction. Annu Rev Biochem 2000, 69:183-215.

36. Clinical and Laboratory Standards Institute: Methods for dilution antimicrobial susceptibility test for bacteria that grow aerobically-approved standard-ninth edition, M07-A9., vol. M07-A9. Wayne PA: CLSI; 2012.

37. Pachon-Ibanez ME, Jimenez-Mejias ME, Pichardo C, Llanos AC, Pachon J: Activity of tigecycline (GAR-936) against Acinetobacter baumannii strains, including those resistant to imipenem. Antimicrob Agents Chemother 2004, 48(11):4479-4481.

38. Sambrook J, Russell W: Molecular cloning: a laboratory manual. 3rd edition. Cold Spring Harbor, NY: Cold Spring Harbor Laboratory Press; 2001.

39. Li J, Rayner CR, Nation RL, Owen RJ, Spelman D, Tan KE, Liolios L: Heteroresistance to colistin in multidrug-resistant Acinetobacter baumannii. Antimicrob Agents Chemother 2006, 50(9):2946-2950.

40. Hoang $\Pi$, Karkhoff-Schweizer RR, Kutchma AJ, Schweizer HP: A broad-host-range Flp-FRT recombination system for site-specific excision of chromosomally-located DNA sequences: application for isolation of unmarked Pseudomonas aeruginosa mutants. Gene 1998, 212(1):77-86.

41. Reuss O, Vik A, Kolter R, Morschhauser J: The SAT1 flipper, an optimized tool for gene disruption in Candida albicans. Gene 2004, 341:119-127.

42. Stynen B, Van Dijck P, Tournu H: A CUG codon adapted two-hybrid system for the pathogenic fungus Candida albicans. Nucleic Acids Res 2010, 38 (19):e184.

43. Hunger M, Schmucker R, Kishan V, Hillen W: Analysis and nucleotide sequence of an origin of DNA replication in Acinetobacter calcoaceticus and its use for Escherichia coli shuttle plasmids. Gene 1990, 87(1):45-51.

44. Liou ML, Soo PC, Ling SR, Kuo HY, Tang CY, Chang KC: The sensor kinase BfmS mediates virulence in Acinetobacter baumannii. J Microbiol Immunol Infect. in press.

45. Hsu PC, Yang CY, Lan CY: Candida albicans Hap43 is a repressor induced under low-iron conditions and is essential for iron-responsive transcriptional regulation and virulence. Eukaryot Cell 2011, 10(2):207-225.

46. Bantar C, Di Chiara M, Nicola F, Relloso S, Smayevsky J: Comparative in vitro bactericidal activity between cefepime and ceftazidime, alone and associated with amikacin, against carbapenem-resistant Pseudomonas aeruginosa strains. Diagn Microbiol Infect Dis 2000, 37(1):41-44.

doi:10.1186/1471-2180-14-119

Cite this article as: Lin et al:: Role of the BaeSR two-component system in the regulation of Acinetobacter baumannii adeAB genes and its correlation with tigecycline susceptibility. BMC Microbiology 2014 14:119.

\section{Submit your next manuscript to BioMed Central and take full advantage of:}

- Convenient online submission

- Thorough peer review

- No space constraints or color figure charges

- Immediate publication on acceptance

- Inclusion in PubMed, CAS, Scopus and Google Scholar

- Research which is freely available for redistribution 